\title{
Modelagem da Polimerização Simultânea de Estireno em Suspensão e Emulsão
}

\author{
Marcelo K. Lenzi, Enrique L. Lima, José Carlos Pinto \\ Programa de Engenharia Química, COPPE, UFRJ
}

\begin{abstract}
Resumo: Apesar dos processos de polimerização em suspensão e emulsão serem processos heterogêneos, cada um origina características peculiares para a distribuição de tamanho de partículas, distribuição de pesos moleculares, taxa de nucleação das partículas de polímero, taxa de polimerização, entre outras. Neste trabalho, são realizadas polimerizações de estireno simultaneamente em suspensão e emulsão. A carga inicial do reator equivale à receita de uma polimerização em suspensão tradicional, enquanto os constituintes da emulsão são adicionados ao longo da batelada. Analisa-se como as propriedades finais do polímero e o curso da polimerização dependem do momento em que a carga característica da emulsão é adicionada à polimerização em suspensão. Apresenta-se, também, um modelo matemático para a descrição do sistema, sendo que o modelo proposto possui boa concordância com dados experimentais de conversão, pesos moleculares médios e curva de distribuição de pesos moleculares. A morfologia da partícula de polímero e os pesos moleculares médios mudam significativamente, dependendo do momento da adição da emulsão, podendo até mesmo ser obtidas curvas de distribuição de pesos moleculares bimodais. Verificou-se que as partículas apresentam características do tipo núcleo/casca, sendo o núcleo formado pelas partículas obtidas pela polimerização em suspensão e a casca formada pelas partículas do processo em emulsão.
\end{abstract}

Palavras-chave: Modelagem, morfologia núcleo/casca, polimerização simultânea, regime semi-batelada.

\section{Modeling Styrene Simultaneous Suspension and Emulsion Polymerization Systems}

Abstract: Although both emulsion and suspension polymerization processes are performed in heterogeneous media, each process presents its own typical characteristics, such as the particle size distribution, molecular weight distribution, polymer particle nucleation rates, rates of polymerization, and so on. In this work styrene polymerizations are carried out in suspension and emulsion processes simultaneously. The initial reactor charge resembles the recipe of standard styrene suspension polymerizations, while emulsion polymerization constituents are added during the batch. The influence of the moment when emulsion feed is started on the course of the polymerization and the effects on the polymer properties are analyzed. A mathematical model is developed to describe the behavior of the system. Simulation data obtained with the proposed model are in good agreement with experimental conversion, average molecular weight and molecular weight distribution data. It was observed that the polymer particle morphology and the average molecular weight change very significantly with the emulsion feed time and that such changes can lead to bimodal molecular weight distributions. Coreshell polymer particles were obtained, with the core being formed by polymer particles from the suspension polymerization process and the shell formed by polymer particles from the emulsion one.

Keywords: Modeling, core-shell, simultaneous polymerization, semi-batch.

\section{Introdução}

As propriedades finais das resinas poliméricas dependem de diversas variáveis, como por exemplo a distribuição de tamanho de partículas, composição do polímero e, principalmente, a forma da curva de distribuição de pesos moleculares (CDPM $)^{[1]}$. Em algumas aplicações específicas CDPMs largas ou bimodais são necessárias, uma vez que a fração de polímero com peso molecular baixo melhora a processabilidade da resina, enquanto a fração de polímero com peso molecular elevado contribui com a melhoria das propriedades mecânicas da resina ${ }^{[2,3]}$.

A literatura apresenta um grande número de técnicas para a produção de resinas com índice de polidispersão elevado $^{[2]}$. No entanto, todas estas técnicas baseiam-se no princípio de misturar diferentes materiais a nível molecular. As principais estratégias são o uso de agentes de transferência de cadeia ${ }^{[4]}$, manipulação das condições operacionais ${ }^{\left[{ }^{[}\right]}$ e o uso de diferentes tipos de catalisadores ${ }^{[6]}$. Além disso, podem ser usados processos de mistura física. Esses processos não são normalmente aconselhados porque o desempenho do produto final pode ser insatisfatório, devido ao menor grau de mistura que se pode conseguir.

Os processos em suspensão se caracterizam pelo fato de um ou mais monômeros insolúveis em um solvente considerado como fase contínua (geralmente usa-se água)

Autor para correspondência: José Carlos Pinto, Programa de Engenharia Química, COPPE, UFRJ, Caixa Postal 68502, CEP: 21945-970, Rio de Janeiro, RJ.E-mail:pinto@peq.coppe.ufrj.br 
estarem dispersos nesta fase através do uso combinado de agentes de suspensão e elevada agitação da mistura. Tipicamente, formam-se partículas com tamanho entre $10 \mu \mathrm{m}-$ $5 \mathrm{~mm}^{[7]}$. A polimerização tem início quando um iniciador solúvel na fase monomérica é adicionado ao sistema. As partículas de monômero são, portanto, o local da reação, podendo cada uma ser considerada como um micro-reator em batelada, operando como um sistema de polimerização em massa ${ }^{[8]}$. O tamanho das partículas poliméricas do produto final está no intervalo $1 \mu \mathrm{m}-10 \mathrm{~mm}$ e a reação se processa normalmente por radicais livres ou via iônica ${ }^{[9]}$. As principais vantagens deste processo são a facilidade de separação do produto final, facilidade de remoção de calor e controle de temperatura, devido ao uso de uma fase contínua ${ }^{[7]}$. Os principais problemas de controle a serem enfrentados nesses sistemas são o controle da distribuição de tamanhos de partícula, normalmente muito heterogênea, e o aumento dos pesos moleculares médios, que são relativamente baixos ${ }^{[10]}$.

Apesar de também serem heterogêneos, processos em emulsão são bastante diferentes dos processos em suspensão. Os sistemas em emulsão são caracterizados por apresentarem uma fase contínua, geralmente água, e pelo uso de surfactante em excesso, que estabilizam as gotas de monômero e servem como principal loco da reação. Normalmente usa-se também um iniciador solúvel na fase contínua, diferentemente dos sistemas em suspensão.

As reações nos sistemas em emulsão podem ser divididas simplificadamente em três intervalos. A nucleação das partículas poliméricas ocorre no primeiro intervalo, quando os radicais formados na fase aquosa ou entram nas micelas (formadas pelo excesso do agente emulsificante) inchadas por monômero (nucleação micelar) ou crescem e eventualmente precipitam (nucleação homogênea). O monômero forma gotas de diâmetro muito elevado (1-10 $\mu \mathrm{m})$ quando comparado ao diâmetro das micelas (da ordem de 1-10nm), de forma que a captura de radicais e conseqüente polimerização nas gotas é pouco provável. Assim, as gotas funcionam como reservatórios de matéria-prima. No segundo intervalo, a polimerização prossegue nas partículas de polímero inchadas com monômero. $\mathrm{O}$ terceiro intervalo tem início quando as gotas de monômero desaparecem. O produto final tem a forma de látex, cujas partículas apresentam tamanho muito menor $(0,1-1,0 \mu \mathrm{m})$ que no caso da polimerização em suspensão ${ }^{[9,11]}$. Dentre outras, as principais vantagens dos processos em emulsão são a possibilidade de se obter elevados pesos moleculares e elevadas taxas de reações simultaneamente, devido à natureza compartimentalizada destes sistemas; facilidade de transferência de calor, devido à natureza heterogênea do meio; maior adequação a normas ambientais e possibilidade de uso do produto final diretamente, dispensando etapa de separação ${ }^{[12]}$. Atualmente sua importância vem crescendo devido à leis ambientais mais rigorosas, pois os processos em emulsão não usam solventes orgânicos ${ }^{[12]}$. Os sistemas em emulsão estão associados fortemente à produção de tintas, adesivos e revestimentos, podendo ser destacados os polímeros derivados de acrilatos. A reação pode ser feita em regime batelada, batelada alimentada (semi-batelada) ou regime contínuo. Os principais problemas de controle estão associados ao controle preciso da curva de distribuição de pesos moleculares do produto final e à manutenção da estabilidade do látex. Além disso, nos casos em que é preciso separar o produto polimérico, a etapa de separação do polímero pode ser limitante.

Materiais com estrutura tipo casca/núcleo têm atraído a atenção nos últimos anos ${ }^{[13]}$. Isto se deve ao fato de o núcleo e a casca apresentarem estruturas diferentes. Geralmente, o material de interesse fica na casca e o núcleo é constituído de um material menos nobre, funcionando como um suporte $^{[14]}$. Deve-se ressaltar que as partículas tipo casca/núcleo possuem propriedades diferentes das partículas constituídas apenas pelo material da casca ou apenas do núcleo. Exemplos sobre a preparação de diversos tipos de partículas com estrutura casca/núcleo, usadas como recheios de colunas cromatográficas, suportes para catalisadores, etc., podem ser encontrados na literatura ${ }^{[15]}$. A principal estratégia usada para preparar essas partículas é a polimerização em duas etapas, geralmente com a separação e purificação do produto final da primeira etapa, para que este possa ser usado como semente da segunda etapa.

Nos sistemas de polimerização apresentados, a determinação de um modelo matemático pode ser uma tarefa complicada devido ao grau de acoplamento das reações, ao número de fases existente no reator, e à ocorrência de fenômenos complexos de transferência de massa e energia entre as fases. A maioria dos trabalhos presentes na literatura leva em conta apenas o cálculo da conversão do monômero e dos pesos moleculares médios, através da consagrada técnica de momentos ${ }^{[16]}$. No entanto, como a forma da curva de distribuição de pesos moleculares determina as propriedades mecânicas da resina polimérica, a modelagem e controle da forma da curva de distribuição de pesos moleculares têm sido o alvo de alguns trabalhos ${ }^{[4]}$.

Verifica-se que diferentes processos de polimerização levam a formação de resinas poliméricas com diferentes características. Uma questão que surge é: por que não combinar estes processos? Por que não usá-los simultaneamente? Sendo assim, este trabalho foca a polimerização simultânea do estireno em suspensão e emulsão com o objetivo de tentar incorporar vantagens de ambos os processos e possivelmente construir uma nova família de produtos. É analisada particularmente a influência que a adição dos componentes típicos de um sistema em emulsão exerce sobre um sistema de polimerização em suspensão. Essa adição pode ser feita de três formas diferentes: na partida da reação, após duas horas de reação (conversões médias) e após quatro horas de reação (elevadas conversões) em suspensão. São apresentados resultados de gravimetria, peso molecular e microscopia óptica que mostram que as características do produto mudam significativamente com o momento de adição dos componentes da emulsão. Além disso, é apresentado um modelo matemático para o sistema, sendo o mesmo usado para descrever a evolução da conversão e dos pesos moleculares médios. Por 


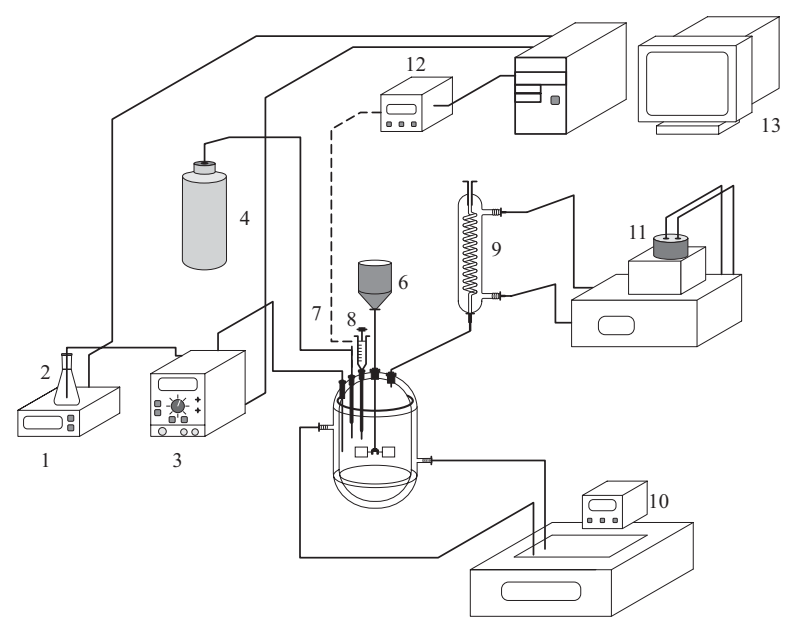

Figura 1. Ilustração do módulo experimental usado. (1) balança (Helmac 1000), (2) Frasco com monômero a ser alimentado, (3) Bomba (Gamma/ Gala Prominent 1000SST), (4) Cilindro de Nitrogênio, (5) Reator de vidro borossilicado com jaqueta e capacidade de 1,0L (FGG Equipamentos Científicos LTDA), (6) Agitador (Fisatom 713-T), (7) Termopar (Tipo: J), (8) Seringa para coleta de amostra, (9) condensador, (10) banho termostático para a jaqueta do reator (Haake DC-3), (11) banho de refrigeração para o condensador (Polyscience KR30-A), (12) chassis para tratamento de sinais adquiridos (National Instruments SCXI-1000), (13) computador para aquisicao de dados (233-MHz Pentium MMX).

fim, deve-se ter em mente que não há nenhuma operação unitária entre as etapas de polimerização que vise a separação da resina polimérica, o que representa uma economia a mais no processo.

\section{Experimental}

O módulo experimental utilizado para a execução dos experimentos é apresentado de forma esquemática na Figura 1. Convém ressaltar que o software para aquisição de dados foi desenvolvido usando-se o Labview versão $5.0^{[17]}$. As placas de conversão analógico-digital e digital-analógico utilizadas são da National Instruments.

Todos os reagentes foram usados sem purificação prévia. Os componentes químicos são água destilada (meio reacional), estireno (monômero, fornecido NITRIFLEX Resinas S/A, com 10 ppm de terc-butil-catecol como inibidor), álcool polivinílico (PVA - agente de suspensão, fornecido por VETEC Química Fina, com grau de pureza superior a 99\%), peróxido de benzoíla (BPO - iniciador solúvel na fase orgânica, fornecido por VETEC Química Fina com 25\% de umidade), lauril sulfato de sódio (SLS - surfactante, fornecido por VETEC Química Fina com grau de pureza superior a $99 \%)$, persulfato de potássio $\left(\mathrm{K}_{2} \mathrm{~S}_{2} \mathrm{O}_{8}\right.$ - inciador solúvel na fase aquosa, fornecido por Merck do Brasil com pureza superior a 99\%), bicarbonato de sódio $\left(\mathrm{NaHCO}_{3}\right.$ - tampão, fornecido por VETEC Química Fina com grau de pureza superior a 99\%). Análises morfológicas foram feitas usando-se um microscópio ótico Stereo Olympus SZH10. A conversão foi determinada gravimetricamente, usando uma estufa com recirculação de ar, cuja temperatura foi mantida aproximadamente constante em $40^{\circ} \mathrm{C}$. Os pesos moleculares mé- dios e a curva de distribuição de pesos moleculares foram determinados com um cromatógrafo (Waters 600E), equipado com quatro colunas (Ultrastyragel), com géis com porosidades $10^{5}, 10^{4}, 10^{3}$ e $500 \AA$ e um detector refratométrico (Waters 410). A calibração deste foi feita usando padrões de poliestireno fornecidos pela Phenomenex ${ }^{\circledR}$ com peso molecular na faixa $10^{3}$ a $2 \times 10^{6} \mathrm{Da}$ e polidispersão menor que 1,05. As análises foram conduzidas com THF grau HPLC, fornecido pela Tedia Company, Inc., e a temperatura de $40{ }^{\circ} \mathrm{C}$.

Os experimentos realizados visaram observar a influência da adição dos componentes de um sistema em emulsão a um sistema em suspensão e foram divididos em três grupos, de acordo com o instante em que a carga do sistema de polimerização em emulsão foi adicionada. No grupo I, a carga da emulsão foi adicionada na partida da reação, em conjunto com a carga do sistema de polimerização em suspensão. No grupo II, a adição se deu duas horas após a partida da reação de polimerização em suspensão e no grupo III, quatro horas após a reação de polimerização em suspensão. No segundo grupo foram realizados ainda experimentos para modificar a curva de distribuição de tamanho de partículas do processo de polimerização em suspensão. Deve-se ressaltar que a carga de emulsão é acompanhada de alimentação de monômero puro durante o processo, para evitar descontrole térmico, com a alimentação mantida a uma vazão constante. A temperatura das reações foi mantida constante em $85^{\circ} \mathrm{C}$. Além disso, os compomentes sólidos sempre foram adicionados sob a forma de solução.

\section{Modelagem matemática}

O modelo matemático para o sistema está baseado em um mecanismo de reação via radicais livres clássico ${ }^{[18]}$. Entretanto, em suspensão considera-se apenas a terminação por combinação como etapa de terminação do processo. Quanto às reações de transferência, considera-se apenas a transferência para monômero.

\begin{tabular}{l|c}
\hline Iniciação & Transferência para Monômero \\
$I^{y} \stackrel{k_{d}}{\longrightarrow} 2 R^{*^{y}}$ & $P_{j}^{*^{y}}+M^{y} \stackrel{k_{t f m}}{\longrightarrow} \Omega_{j}^{y}+P_{1}^{*^{y}}$ \\
$R^{*^{y}}+M^{y} \longrightarrow P_{1}^{*^{y}}$ & \\
\hline Propagação & Terminação por Combinação \\
$P_{j}^{*^{y}}+M^{y} \stackrel{k_{p}}{\longrightarrow} P_{j+1}^{\bullet^{y}}$ & $P_{j}^{*^{y}}+P_{k}^{*^{y}} \stackrel{k_{t c o m b}}{\longrightarrow} \Omega_{j+k}^{y}$ \\
\hline
\end{tabular}

A derivação do modelo matemático para o sistema de polimerização simultâneo está baseado na existência de 5 fases no reator. A fase I representa as partículas de polímero em suspensão; a fase II representa as partículas de polímero em emulsão; a fase III está associada ao polímero que recobre as partículas de suspensão, a fase IV são as gotas de monômero existentes no reator e a fase $\mathrm{V}$ é a fase aquosa.

O modelo é composto por um conjunto de equações algébrico-diferenciais, obtidas a partir de balanços de mas- 
sa para os constituintes do sistema em todas as fases, admitindo-se regime semi-batelada. O processo em suspensão apresenta as taxas de polimerização de um mecanismo via radicais livres clássico, sendo dadas por:

$$
\begin{aligned}
& r_{p}^{y}=k_{p} \cdot\left[M^{y}\right] \cdot\left[P^{\bullet^{y}}\right] \\
& {\left[P^{\bullet y}\right]=\sqrt{\frac{2 \cdot \mathrm{f}^{\mathrm{y}} \cdot \mathrm{k}_{\mathrm{d}}^{\mathrm{y}} \cdot\left[\mathrm{I}^{\mathrm{y}}\right]}{\mathrm{k}_{\mathrm{tcomb}}}}}
\end{aligned}
$$

Considerou-se o processo de polimerização em emulsão como possuindo caráter de compartimentalização, sendo a taxa de propagação dada por:

$$
\mathrm{r}_{\mathrm{P}}^{\mathrm{y}}=\mathrm{k}_{\mathrm{P}} \cdot\left[\mathrm{M}^{\mathrm{Y}}\right] \cdot \overline{\mathrm{n}} \cdot \mathrm{N}_{\mathrm{P}}
$$

Como o monômero usado é o estireno, pode-se usar um dos resultados clássicos de Smith-Ewart ${ }^{[19]}$, o qual apresenta o número médio de radicais por partícula $(\bar{n})$ como sendo igual 0,5. A terminação ocorre quando um radical da fase aquosa entra em uma partícula que contenha um radical em crescimento. A expressão da taxa de formação de partículas na polimerização em emulsão é dada por:

$$
\frac{\mathrm{d} \mathrm{N}_{\mathrm{P}}}{\mathrm{dt}}=\left(2 \cdot \mathrm{f}^{\mathrm{y}} \cdot \mathrm{k}_{\mathrm{d}}^{\mathrm{y}} \cdot \mathrm{I}^{\mathrm{y}}\right) \cdot\left[\frac{\mathrm{N}_{\text {mic }}}{\mathrm{N}_{\text {mic }}+\mathrm{N}_{\mathrm{P}}}\right]-\mathrm{k}_{\mathrm{NP}} \cdot \mathrm{N}_{\mathrm{P}}
$$

$\mathrm{Na}$ equação acima, o primeiro termo indica a quantidade de radicais existentes na fase aquosa. O segundo termo mostra a probabilidade de um dado radical entrar em uma micela, ao invés de entrar em uma partícula pré-existente. O terceiro termo indica a coalescência das partículas de emulsão sobre as partículas de suspensão, para que haja formação da estrutura casca-núcleo. Considerou-se, para fins de modelagem, que todas as perdas de material da fase II (polimerização em emulsão) para fase III (revestimento polimérico) como sendo de primeira ordem, dependendo do termo $\mathrm{k}_{\mathrm{NP}}$. Essa hipótese é compatível com a idéia de que o número de partículas características do processo em emulsão é muito maior que o número de partículas características do processo em suspensão. Para calcular o número de micelas, admite-se que o emulsificante tende primeiro a saturar a água; em seguida, a recobrir as partículas existentes; e, por fim, a quantidade restante é responsável pela formação das micelas. As trocas de massa entre as fases não são consideradas instantâneas, sendo necessária a estimação dos coeficientes de transferência.

Os pesos moleculares ponderal médio $(\mathrm{Mw})$ e numérico médio (Mn) são calculados usando-se a técnica dos momentos $^{[16]}$. Deve-se obter o balanço populacional dos radicais em crescimento $\left(P_{i}^{*}\right)$ e das cadeias de polímero morto $\left(\Omega_{\mathrm{i}}\right)$ para as fases I, II e III. Em seguida, usando-se as Equações (05) e (06), que representam o momento de ordem $\mathrm{k}$ do polímero morto e radicais em crescimento na fase y $(\mathrm{y}=\mathrm{I}$,II, III) respectivamente, determina-se um conjunto de equações para descrever estes momentos para estas fases.

$$
\begin{aligned}
& Q_{k}^{y}=\sum_{i=1}^{\infty} n^{k} \cdot \Omega_{i}^{y} \\
& \mu_{\mathrm{k}}^{\mathrm{y}}=\sum_{\mathrm{i}=1}^{\infty} \mathrm{n}^{\mathrm{k}} \cdot \mathrm{P}_{\mathrm{i}}^{\bullet \mathrm{y}}
\end{aligned}
$$

Para os radicais vivos é possível admitir a hipótese de estado pseudo-estacionário ${ }^{[16]}$. As Equações (07) e (08) ilustram as equações para o cálculo de $M_{w}$ e $M_{n}$. As curvas de distribuição de pesos moleculares foram calculadas de acordo com a técnica apresentada por ${ }^{[20]}$ também baseada na hipótese do estado pseudo-estacionário para os radicais em crescimento.

$$
\begin{aligned}
M_{w}= & \frac{\sum_{y} Q_{2}^{y}}{\sum_{y} Q_{1}^{y}} \cdot P M_{m o n} \\
M_{n}= & \frac{\sum_{y} Q_{1}^{y}}{\sum_{y} Q_{0}^{y}} \cdot P M_{\text {mon }}
\end{aligned}
$$

Uma última hipótese a ser considerada na derivação do modelo matemático está associada ao fato de que o material da fase II (emulsão), que passa a recobrir as partículas de suspensão para originar a fase III, perde a característica de compartimentalização, passando a se comportar similarmente ao processo em suspensão. Para a resolução do modelo matemático usou-se o pacote de integração DASSL ${ }^{[21]}$. As constantes cinéticas foram obtidas da literatura ${ }^{[18]}$. Como será visto na próxima seção, $\mathrm{k}_{\mathrm{NP}}$, foi determinado a partir dos dados experimentais obtidos neste estudo.

\section{Resultados e Discussão}

Serão apresentados apenas os resultados experimentais referentes ao grupo II (experimentos REAC1 e REAC2) e ao grupo III (experimento REAC3). Resultados referentes ao grupo I podem ser encontrados em ${ }^{[22]}$. As receitas e as condições operacionais dos experimentos são apresentadas na Tabela 1 .

As reações do grupo II são caracterizadas pelo fato de que no instante inicial da reação estão presentes apenas os componentes do sistema de polimerização em suspensão, sendo que após decorridas duas horas de reação, as partículas de polímero formadas apresentam conversões intermediárias. Neste instante, são adicionados os componentes do sistema de polimerização em emulsão, fazendo com que ambos operem simultaneamente no mesmo reator. A diferença fundamental entre os experimentos REAC1 e REAC2 está associada às condições operacionais, mais especificamente à velocidade de agitação e a forma do impelidor usado. O objetivo desta diferença foi produzir polímero em suspensão com tamanho de partícula diferente, para que pudesse ser analisada a influência 
Tabela 1. Receita das Polimerizações ${ }^{[1]}$

\begin{tabular}{|c|c|c|c|}
\hline \multicolumn{4}{|c|}{ Receita da Polimerização } \\
\hline Componentes - $[\mathrm{g}]$ & REAC1 & REAC2 & REAC3 \\
\hline Estireno & $\begin{array}{c}200,0(100,0 / 30,0-70,0) \\
\text { alim : 45,0 g }\end{array}$ & $\begin{array}{c}200,0(100,0 / 30,0-70,0) \\
\text { alim : 45,0 g }\end{array}$ & $\begin{array}{c}200,0(100,0 / 30,0-70,0) \\
\text { alim : 60,0 g }\end{array}$ \\
\hline Água & $485,0(370,0 / 115,0)$ & $485,0(370,0 / 115,0)$ & $512,0(400,0 / 115,0)$ \\
\hline SLS & 2,50 & 2,50 & 2,50 \\
\hline $\mathrm{NaHCO}_{3}$ & 0,30 & 0,30 & 0,30 \\
\hline $\mathrm{K}_{2} \mathrm{~S}_{2} \mathrm{O}_{8}$ & 0,30 & 0,30 & 0,30 \\
\hline PVA & 3,00 & 2,00 & 3,00 \\
\hline $\mathrm{BPO}$ & 4,00 & 4,00 & 4,00 \\
\hline \multicolumn{4}{|c|}{ Condições Operacionais da Polimerização } \\
\hline Componentes & REAC1 & REAC2 & REAC3 \\
\hline Temperatura $-\left[{ }^{\circ} \mathrm{C}\right]$ & 85 & 85 & 85 \\
\hline Agitação - [rpm] & $1500 \pm 50$ & $1080 \pm 50$ & $1000 \pm 50$ \\
\hline Impelidor & 01 & 02 & 01 \\
\hline
\end{tabular}

${ }^{1} \mathrm{Na}$ linha referente ao Estireno, o valor fora dos parênteses indica a quantidade total de estireno da receita. Dentro dos parênteses, é indicada a quantidade de estireno correspondente à receita da polimerização em suspensão (adicionada ao reator na partida da reação) e à da polimerização em emulsão, sendo os valores adicionados junto com os componentes e pelo sistema de bombeamento, respectivamente. $\mathrm{O}$ termo alim indica a quantidade de estireno efetivamente adicionada ao sistema reacional. Na linha referente à água, o valor fora do parênteses indica a quantidade total de água da receita, enquanto a quantidade dentro dos parênteses indica a quantidade de água correspondente à receita da polimerização em suspensão (adicionada ao reator na partida da reação) e à da polimerização em emulsão (sendo adicionada junto com os componentes), respectivamente. O impelidor tipo 01 apresenta 6 lâminas com ângulo de $45^{\circ}$ entre cada uma e o tipo 02 tem a forma de impelidor naval.

desta variável sobre o termo $\mathrm{k}_{\mathrm{NP}}$ presente no modelo matemático proposto. As reações do grupo III, representado pelo experimento REAC3, por outro lado teve por principal objetivo a variação da conversão das partículas do processo de polimerização em suspensão e a avaliação desta alteração sobre as propriedades da resina polimérica final.

Uma análise de tamanho das partículas imediatamente antes da adição dos componentes do processo de polimerização em emulsão revelou um diâmetro médio das partículas referente à REAC1 de aproximadamente $49 \mu \mathrm{m}$. Para REAC2, o tamanho médio das partículas neste mesmo instante é de $550 \mu \mathrm{m}$, enquanto para REAC 3 o tamanho médio das partículas também neste mesmo instante é de $65,5 \mu \mathrm{m}$. As Figuras 2a, 2b e 2c apresentam as partículas de polímero formadas pelo processo em suspensão, imediatamente antes da adição dos componentes do sistema de polimerização em emulsão, para REAC1, REAC2 e REAC3, respectivamente. Observa-se, após a secagem, pérolas características do sistema em suspensão.

As micrografias apresentadas nas Figuras $2 \mathrm{~d}, 2 \mathrm{e}$ e $2 \mathrm{f}$ referem-se ao produto final das reações, REAC1, REAC2 e REAC3 respectivamente. Observa-se um recobrimento completo das partículas de suspensão. Constatou-se que a técnica de polimerização simultânea apresenta uma eficiência de coleta muito grande, o que pode ser confirmado pelo fato de que o sobrenadante de REAC1 apresentou um teor de sólidos de apenas $0,4 \%$ para REAC1, 2,0\% para REAC2, e 1,4\% para REAC3 ao mesmo tempo que a análise do tamanho das partículas por espalhamento de luz deste mesmo sobrenadante indicou um sinal ruidoso e fraco, característico da baixa concentração de partículas em emulsão. Observase ainda que a espessura do recobrimento das partículas no experimento REAC3 é menor do que nos experimentos REAC1 e REAC2, provavelmente devido à menor conversão do monômero apresentada, fazendo com que as partículas em REAC1 e REAC2 estivessem mais pegajosas. Por fim, a Figura $2 \mathrm{~g}$ mostra com clareza o núcleo, formado pela partícula do processo em suspensão, e a casca, formada pela coalescência das partículas do processo em emulsão.

Como apresentado anteriormente, o modelo matemático que descreve o sistema de polimerização simultânea possui um termo de coalescência, denominado $\mathrm{k}_{\mathrm{NP}}$. Este controla a taxa de recobrimento das partículas poliméricas existentes no reator após a adição dos componentes do sistema de polimerização em emulsão. Observa-se que este termo é função de duas variáveis principais: i) tamanho das partículas formadas pela polimerização em suspensão, ii) grau de conversão das partículas formadas pela polimerização em sus- 


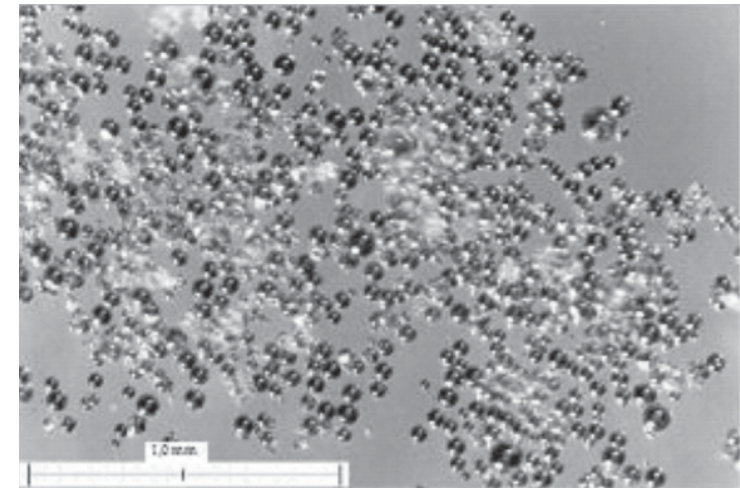

Figura 2a. Micrografia de amostra de REAC1 no instante $\mathrm{t}=120 \mathrm{~min}-70 \mathrm{x}$

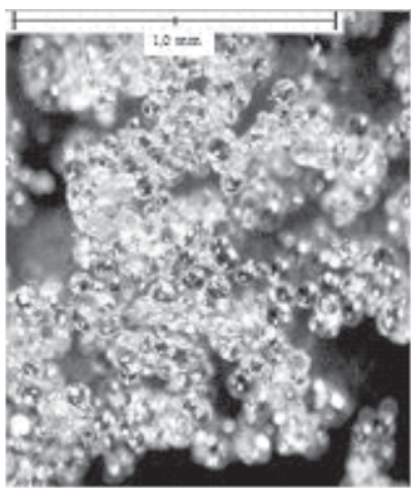

Figura 2c. Micrografia de amostra de REAC3 no instante $t=240 \mathrm{~min}-50 \mathrm{x}$

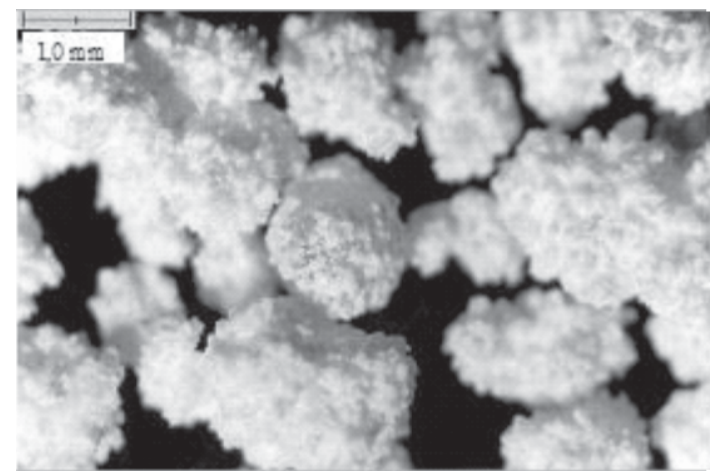

Figura 2e. Micrografia de amostra de REAC2 no instante $\mathrm{t}=240 \mathrm{~min}-25 \mathrm{x}$

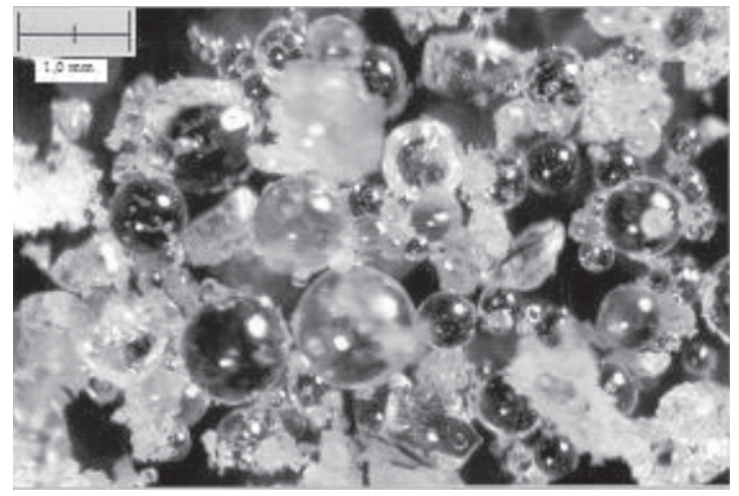

Figura 2b. Micrografia de amostra de REAC2 no instante $t=120 \mathrm{~min}-25 \mathrm{x}$

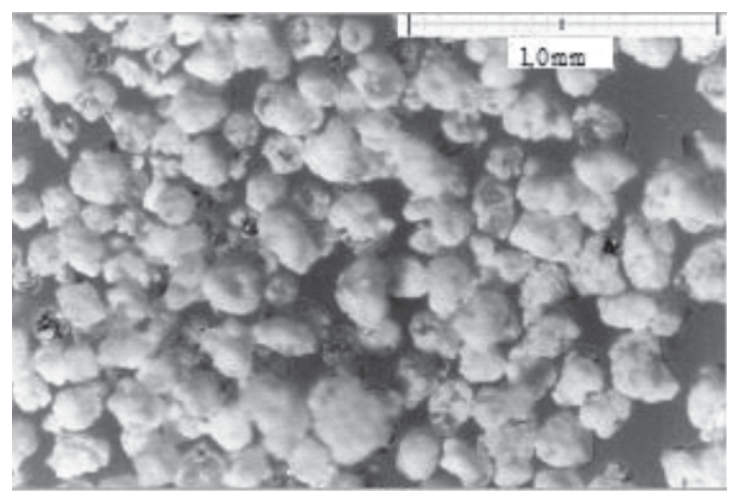

Figura 2d. Micrografia de amostra de REAC1 no instante $t=360 \min -70 x$

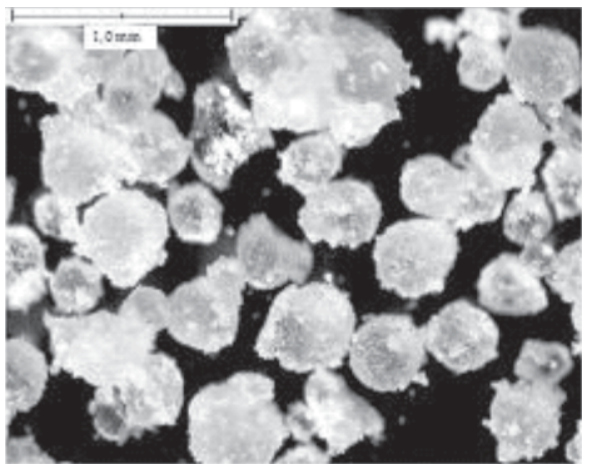

Figura 2f. Micrografia de amostra de REAC3 no instante $\mathrm{t}=520 \mathrm{~min}-50 \mathrm{x}$

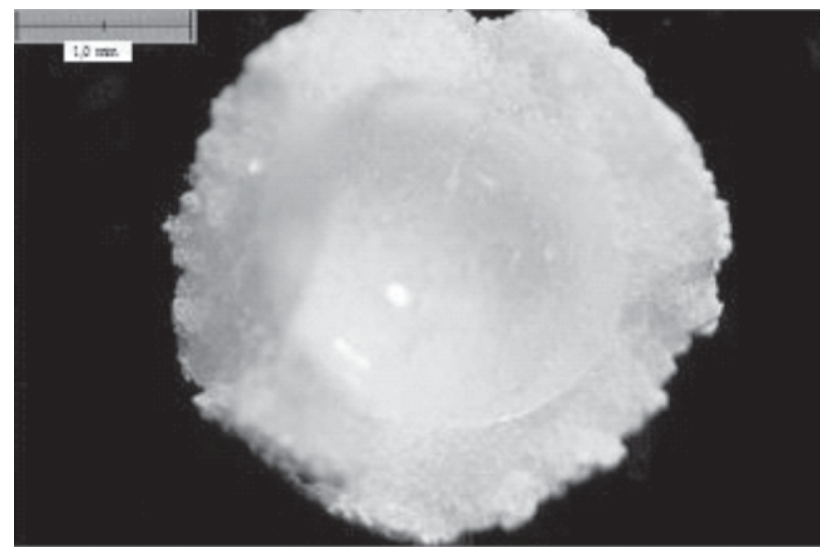

Figura 2g. Micrografia de amostra de REAC2 no instante $t=240 \min -35 x$ 
pensão. Para o desenvolvimento do modelo matemático, optou-se por considerar o termo $\mathrm{k}_{\mathrm{NP}}$ como uma função degrau, como dado pela Equação (09). Para o experimento REAC3, no entanto, deve ser considerado ainda as duas horas a mais que o processo de polimerização em suspensão ocorre antes da adição da carga da emulsão. Com isso, considera-se que o degrau ocorre no tempo de reação igual a 330 min.

$$
k_{N P}= \begin{cases}k_{N P 0} & , t \leq 190 \mathrm{~min} \\ 10^{-6} & , t>190 \mathrm{~min}\end{cases}
$$

Após as análises de micrografia, verificou-se que o grau de recobrimento variava bastante nos instantes iniciais, após a adição dos componentes do sistema de polimerização em emulsão. No entanto, após um dado tempo de reação as alterações na morfologia da partícula não eram mais perceptíveis. Os valores de $\mathrm{k}_{\mathrm{NPo}}$ ajustados para os experimentos REAC1 e REAC2, a partir dos dados experimentais coletados, foram 2,00 e 0,01 respectivamente. Verifica-se que o valor obtido para REAC1 é muito maior do que o encontrado para REAC2, o que está de acordo com as observações anteriores e mostra que a eficiência de coleta depende da distribuição de tamanhos de partículas suspensas. A influência da conversão neste caso é minimizada porque a conversão do monômero é aproximadamente a mesma, enquanto o diâmetro da partícula formada pelo processo de polimerização em suspensão é maior em REAC2 (comparar Figuras 2a e 2b), devido ao uso de sistema de agitação e velocidade de agitação diferentes.

Por outro lado, parece bastante claro que $\mathrm{k}_{\mathrm{NP}}$ depende do estado da partícula polimérica em suspensão. Para REAC3, o valor de $\mathrm{k}_{\mathrm{NPo}}$ ajustado foi equivalente à $2,0 \times 10^{-4}$, bastante inferior aos valores apresentados anteriormente. Observa-se que o diâmetro médio das partículas de REAC3 é intermediário ao diâmetro médio de REAC2 e REAC1. Assim, o valor de $\mathrm{k}_{\mathrm{NPo}}$ para REAC3 deveria ser intermediário aos de REAC2 e REAC1, caso o estado da partícula não tivesse influência. No entanto, o valor obtido para $\mathrm{k}_{\mathrm{NPo}}$ é menor que o de REAC1, o que indica, de forma inequívoca, que a taxa de aglomeração (coalescência) depende do estado da partícula.

A Figura 3a apresenta os resultados de conversão obtidos para o experimento REAC2. A reta vertical indica o instante em que os componentes do sistema de polimerização em emulsão foram adicionados ao sistema. Verifica-se uma boa concordância entre os dados experimentais e o modelo proposto para ambas as etapas da reação. Deve-se salientar que as conversões máximas são controladas pela polimerização em suspensão, dado que o material produzido em emulsão é coletado do meio com grande eficiência, sendo este fato um indicativo adicional de interação entre os processos de suspensão e emulsão. A Figura $3 \mathrm{~b}$ apresenta a evolução dos pesos moleculares médios do experimento REAC2. Verifica-se que há um aumento tanto no valor do peso molecular quanto no valor do índice de polidispersão após a adição dos componentes do sistema de polimerização em emulsão, o que já era esperado, tendo-se em vista as características deste processo. Por fim, a Figura $3 \mathrm{c}$ compara valores teóricos e experimentais para a curva de distribuição de pesos moleculares no instante $t=180$ min, ou seja, após 1 hora de reação simultânea.

A despeito da complexidade do sistema, o ajuste dos dados experimentais pode ser considerado excelente. Analisandose a Figura 3c, observa-se um ligeiro desvio na região de pesos moleculares elevados, apesar do resultado ser considerado satisfatório. Isto ocorre provavelmente por causa da aproximação usada para $\mathrm{k}_{\mathrm{NP}}$. Deve-se ressaltar que foi usada uma

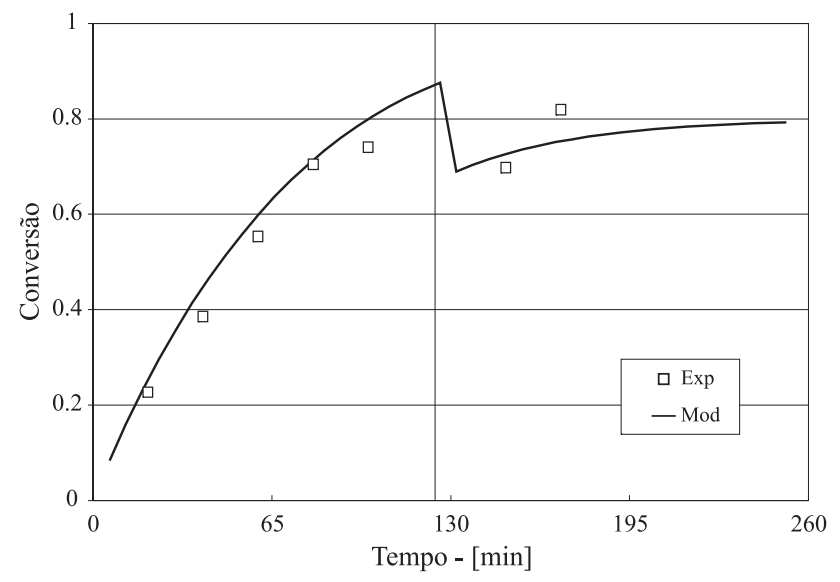

Figura 3a. Comparação entre dados experimentais (Exp) e de simulação (Mod) referentes à conversão apresentada pelo experimento REAC2.

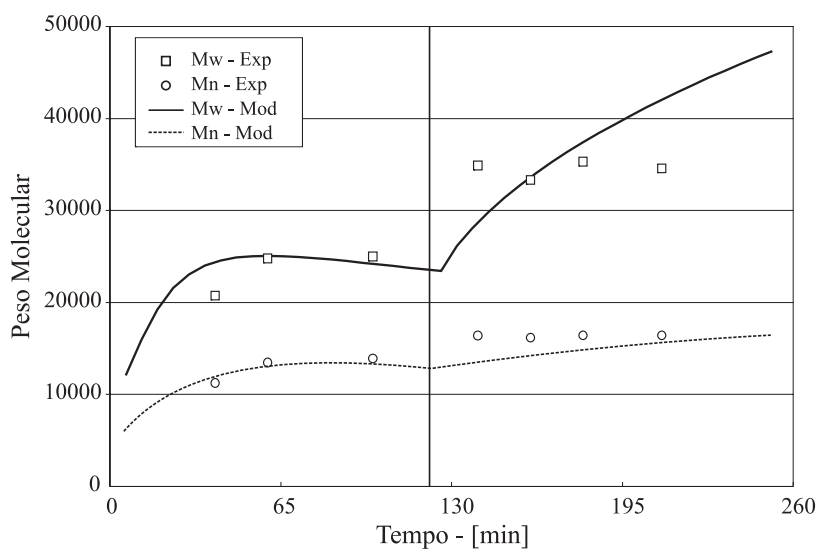

Figura 3b. Comparação entre dados experimentais (Exp) e de simulação (Mod) referentes à evolução dos pesos moleculares médios $\left(\mathrm{M}_{\mathrm{w}}\right.$ e $\left.\mathrm{M}_{\mathrm{n}}\right)$ do experimento REAC2

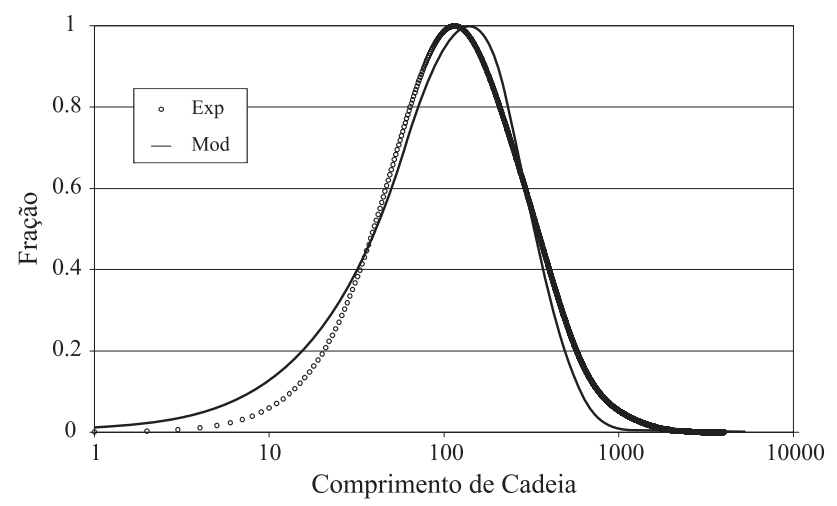

Figura 3c. Comparação entre a distribuição de pesos moleculares (MWD) experimental e a prevista pelo modelo para REAC2 no instante $\mathrm{t}=180 \mathrm{~min}$ 
função degrau para representá-lo, embora $\mathrm{k}_{\mathrm{NP}}$ deva ser uma função contínua do estado da partícula. Além disso, deve-se também ter em mente que, por se tratar de um sistema de polimerização simultâneo, no qual os sistemas em operação produzem materiais com morfologia e pesos moleculares médios diferentes, erros de amostragem, inevitáveis, podem também contribuir com algumas imprecisões.

A Figura 4 apresenta os resultados referentes ao experi-

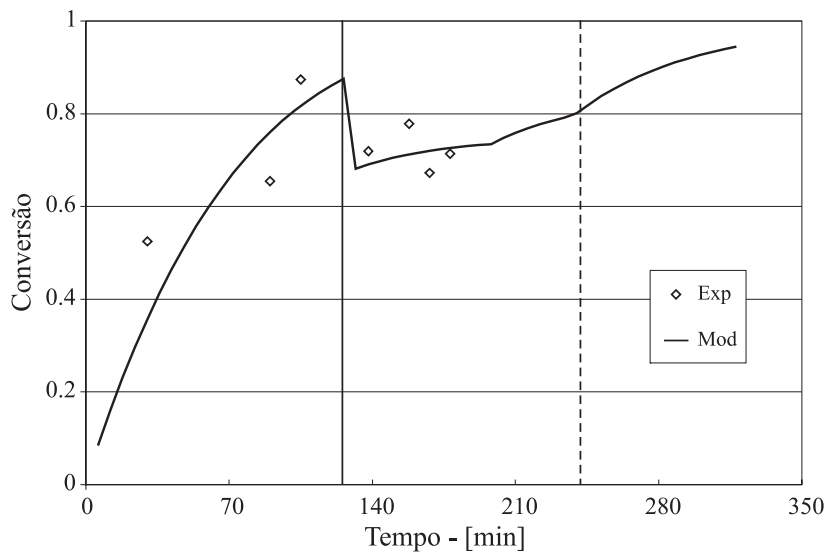

Figura 4a. Comparação entre dados experimentais (Exp) e de simulação (Mod) referentes à conversão apresentada pelo experimento REAC1

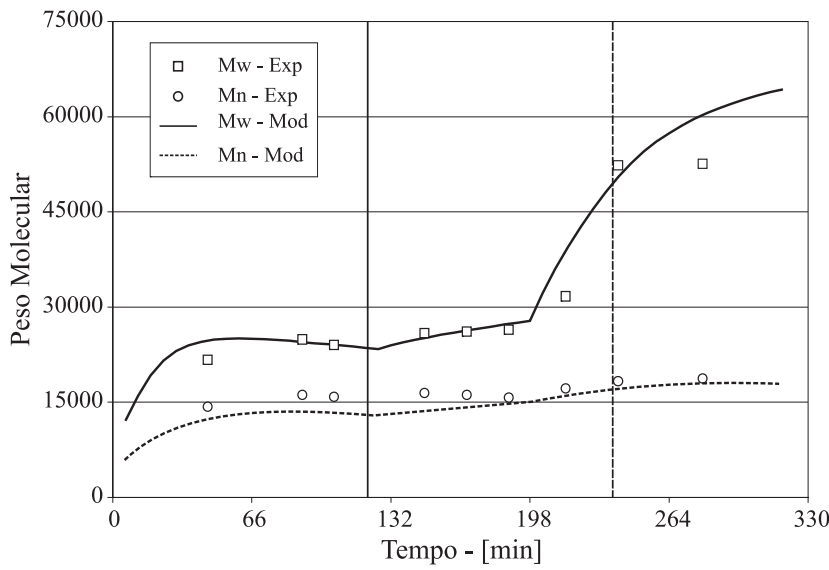

Figura 4b. Comparação entre dados experimentais (Exp) e de simulação (Mod) referentes à evolução dos pesos moleculares médios $\left(\mathrm{M}_{\mathrm{w}}\right.$ e $\left.\mathrm{M}_{\mathrm{n}}\right)$ do experimento REAC1

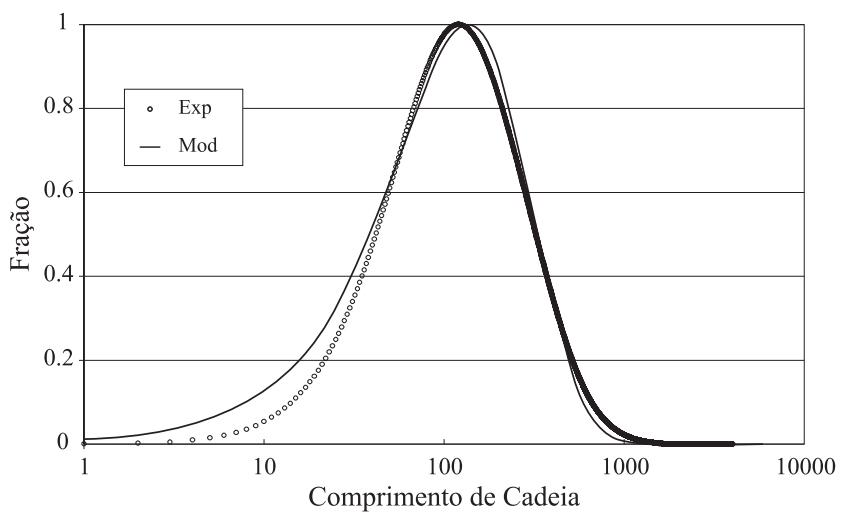

Figura 4c. Comparação entre a distribuição de pesos moleculares (MWD) experimental e a prevista pelo modelo para REAC1 no instante $\mathrm{t}=168 \mathrm{~min}$ mento REAC1, visando a comparação entre resultados experimentais e teóricos obtidos a partir do modelo matemático apresentado. A Figura 4a compara resultados teóricos e experimentais relacionados à conversão do monômero. A reta vertical cheia indica o instante em que os componentes do sistema de polimerização em emulsão foram adicionados ao sistema. A reta vertical tracejada indica o momento em que houve o corte na alimentação de monômero.

Observa-se uma boa concordância entre os resultados experimentais e as previsões do modelo matemático. A Figura $4 \mathrm{~b}$ apresenta a evolução dos pesos moleculares médios do experimento REAC1. Observa-se, como esperado, tanto um aumento no valor do peso molecular quanto no valor do índice de polidispersão a partir da adição dos componentes do sistema de polimerização em emulsão. Este aumento prossegue mesmo com o corte da vazão de alimentação. A qualidade do ajuste pode ser considerada excelente. A Figura $4 c$ compara a MWD experimental e a MWD obtida pelo modelo para a amostra de polímero retirada no instante $\mathrm{t}=168 \mathrm{~min}$; ou seja, quando havia aproximadamente 50 minutos de reação simultânea em suspensão e emulsão.

A Figura 5a apresenta a comparação entre os resultados experimentais e previstos pelo modelo para a conversão, com relação ao experimento REAC3, observando-se uma boa concordância. A Figura $5 b$ apresenta a evolução dos pesos moleculares médios. Novamente, ressalta-se o aumento no valor do índice de polidispersão da resina polimérica após a adição dos componentes do sistema de polimerização em emulsão. Observa-se que o modelo apresenta a mesma tendência dos resultados experimentais, embora o ajuste apresente alguns desvios para pesos moleculares mais elevados. Novamente, deve-se ressaltar que a escolha de um outro tipo de função para $\mathrm{k}_{\mathrm{NP}}$ pode vir a melhorar o ajuste. Além disso, o modelo usado não é perfeito e para essas reações simultâneas, erros de amostragem podem originar grandes desvios nas análises de peso molecular. Contudo, em REAC3 os valores $\mathrm{M}_{\mathrm{w}}$ e IP indicam que ocorre uma maior influência do polímero produzido em emulsão que em REAC3, o que também confirma menores valores de $\mathrm{k}_{\mathrm{NP}}$ observados. A Figura $5 \mathrm{c}$ apresenta a curva de distribuição de pesos moleculares para o polímero no instante $\mathrm{t}=280 \mathrm{~min}$, ou seja, com $40 \mathrm{minu}-$ tos de reação simultânea. Observa-se que a MWD experimental é menos aberta do que a prevista pelo modelo, como também ilustrado na Figura 5 b, tendo em vista a distância entre os valores de $\mathrm{M}_{\mathrm{W}}$ e $\mathrm{M}_{\mathrm{N}}$.

\section{Conclusões}

Verifica-se que é possível realizar a polimerização simultânea de estireno em suspensão e emulsão e incorporar ao produto final características particulares de cada processo. Além disso, validou-se com dados experimentais um modelo matemático para descrição do sistema. Entretanto, o instante de adição dos componentes da emulsão parece ser de fundamental importância no processo, afetando particularmente as taxas de reação. Verificou-se que esta técnica, a polimerização simultânea, pode ser usada no recobrimento de partículas e para a produção de estruturas tipo casca/núcleo. Além disso, e que a 


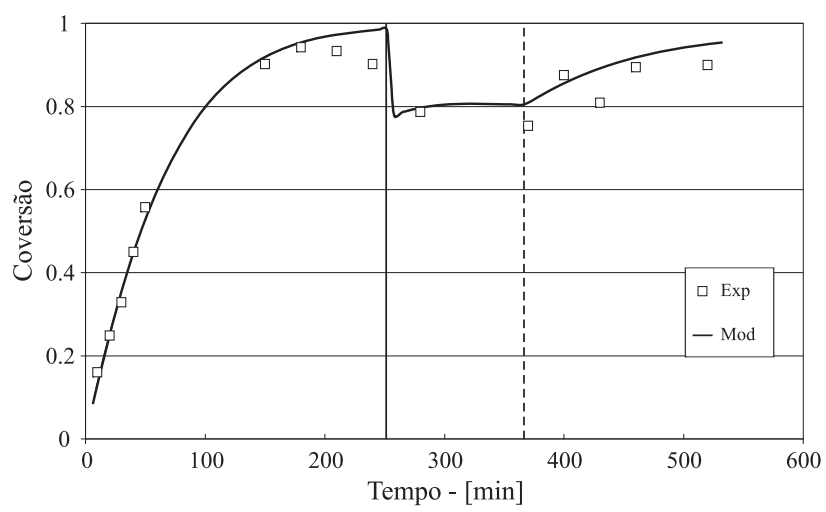

Figura 5a. Comparação entre dados experimentais (Exp) e de simulação (Mod) referentes à conversão apresentada pelo experimento REAC3

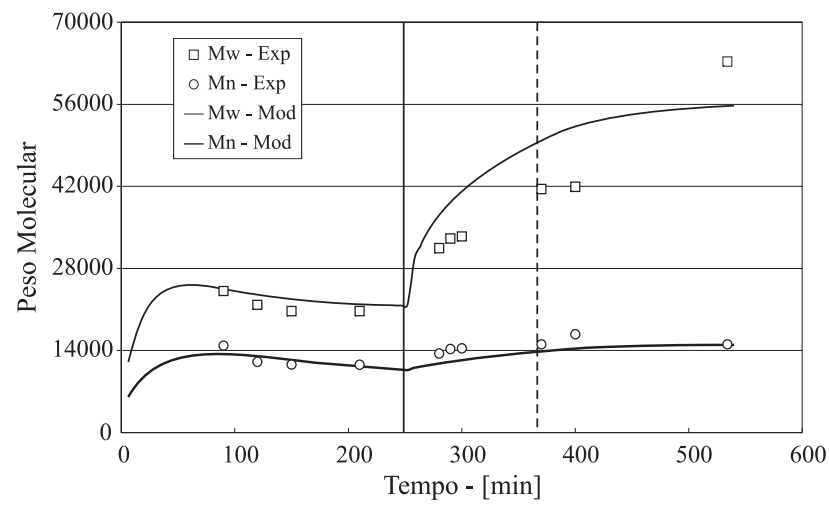

Figura 5b. Comparação entre dados experimentais (Exp) e de simulação (Mod) referentes à evolução dos pesos moleculares médios $\left(M_{w}\right.$ e $\left.M_{n}\right)$ do experimento REAC3

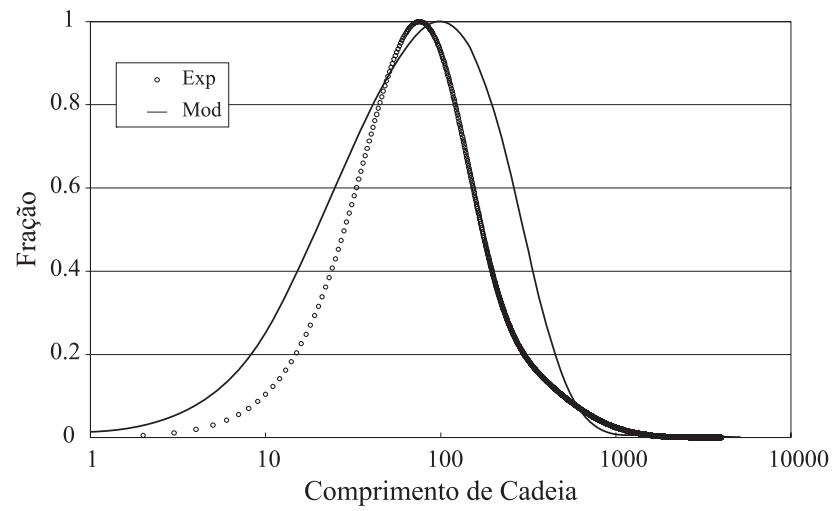

Figura 5c. Comparação entre a distribuição de pesos moleculares (MWD) experimental e a prevista pelo modelo para REAC3 no instante $\mathrm{t}=280 \mathrm{mi}$

taxa de recobrimento, variável de suma importância para o modelo matemático, depende fundamentalmente de duas variáveis: conversão do monômero e tamanho das partículas do sistema de polimerização em suspensão existente no reator.

\section{Agradecimentos}

Os autores agradecem à CAPES e ao CNPq pelo apoio financeiro e pelas bolsas de pesquisa. Os autores também agradecem à NITRIFLEX S/A pela doação de reagentes.

\section{Nomenclatura}

\begin{tabular}{|c|c|}
\hline $\mathrm{f}^{\mathrm{y}}$ & Eficiência do iniciador na fase y \\
\hline $\mathrm{i}$ & Comprimento de cadeia \\
\hline $\mathrm{I}^{\mathrm{y}}$ & Iniciador na fase $y$ \\
\hline$\left[\mathrm{I}^{\mathrm{y}}\right]$ & Concentração de iniciador na fase $\mathrm{y}-\left[\mathrm{mol} / \mathrm{cm}^{3}\right]$ \\
\hline${ }_{\mathrm{d}}^{\mathrm{k}}$ & Constante de decomposição do iniciador - $[1 / \mathrm{s}]$ \\
\hline $\mathrm{k}_{\mathrm{p}}$ & Constante de propagação $-\left[\mathrm{cm}^{3} /(\right.$ molxs $\left.)\right]$ \\
\hline $\mathrm{k}_{\text {tcomb }}$ & Constante de terminação por combinação $-\left[\mathrm{cm}^{3} /(\operatorname{mol} \times s)\right]$ \\
\hline $\mathrm{k}_{\mathrm{NP}}$ & Parâmetro do processo de coalescência \\
\hline $\mathrm{k}_{\mathrm{tfm}}$ & $\begin{array}{l}\text { Constante de transferência de cadeia para o monômero - } \\
{\left[\mathrm{cm}^{3} /(\text { mol } \times \mathrm{s})\right]}\end{array}$ \\
\hline $\mathrm{M}^{\mathrm{y}}$ & Monômero na fase y \\
\hline$\left[\mathrm{M}^{\mathrm{y}}\right]$ & Concentração de monômero na fase $\mathrm{y}-\left[\mathrm{mol} / \mathrm{cm}^{3}\right]$ \\
\hline $\mathrm{M}_{\mathrm{w}}$ & Peso molecular ponderal médio - [g/gmol] \\
\hline $\mathrm{M}_{\mathrm{n}}$ & Peso molecular numérico médio - [g/gmol] \\
\hline $\bar{n}$ & $\begin{array}{l}\text { Número médio de radicais por partícula na polimerização } \\
\text { em emulsão }\end{array}$ \\
\hline $\mathrm{N}_{\text {mic }}$ & Número de micelas \\
\hline $\mathrm{N}_{\mathrm{p}}$ & Número total de partículas na polimerização em emulsão \\
\hline$\left[P^{\bullet y}\right]$ & Concentração total de radicais na fase y - $\left[\mathrm{mol} / \mathrm{cm}^{3}\right]$ \\
\hline$P_{i}^{\bullet y}$ & $\begin{array}{l}\text { Radical com cadeia de comprimento i em crescimento na } \\
\text { fase y }\end{array}$ \\
\hline $\mathrm{PM}_{\text {mon }}$ & Peso molecular do monômero - [g/gmol] \\
\hline$Q_{k}^{y}$ & Momento de ordem $\mathrm{k}$ do polímero morto na fase $\mathrm{y}$ \\
\hline$R^{\bullet y}$ & Radical na fase y \\
\hline$r_{p}^{y}$ & Taxa de propagação da fase $y-\left[\mathrm{mol} /\left(\mathrm{cm}^{3} \times \mathrm{s}\right)\right]$ \\
\hline $\mathrm{t}$ & tempo $-[\mathrm{s}]$ \\
\hline $\mathrm{y}$ & $\begin{array}{l}\text { Fase do sistema de polimerização (I: polimerização em } \\
\text { suspensão, II : olimerização em emulsão, III: recobrimento } \\
\text { polimérico da partícula do processo de polimerização em } \\
\text { suspensão) }\end{array}$ \\
\hline$\Omega_{i}^{y}$ & Polímero morto com cadeia de comprimento i na fase y \\
\hline$\mu_{k}^{y}$ & Momento de ordem $\mathrm{k}$ dos radicais vivos na fase $\mathrm{y}$ \\
\hline
\end{tabular}

\section{Referências Bibliográficas}

1. Latado,A.; Embiruçu,M.; Neto, A.G.M.; Pinto,J.C. Polym. Testing. 20, p.419 (2001).

2. Nele,M.; Pinto,J.C. Macromol. Theory and Simul.. 11, p. 293 (2002). 
3. Bartus,J.; Janules,R.; Boswell,J.; Clark, J. US. Patent. $\mathrm{N}^{\circ} 6.258 .504$ (2001).

4. Vicente,M.; Leiza,J.R.; Asua,J.M. Aiche J.. 47, p. 1594 (2001).

5. Maschio,G.; Bello,T.; Scali,C. Chem. Eng. Sci. 49, p. 5071 (1994).

6. Soares,J.B.P.; Kim,J.D. J. Pol. Sci. Part A: Pol. Chem. 38, p. $1408(2000)$.

7. Vivaldo-Lima,E.; Wood,P.E.; Hamielec,A.E.; Penlidis,A. Ind. Eng. Chem. Res. 36, p. 939 (1997).

8. Dotson,N.A.; Galván,R.; Laurence,R.L.; Tirrel,M. "Polymerization Process Modeling". VCH, New York (1996).

9. Kiparissides,C. Chem. Eng. Sci., 51, p. 1637 (1996).

10. Machado,R.A.F.; Pinto,J.C.; P.H.H. Araújo,P.H.H.; Bolzan, A. Braz. J. Chem. Eng., 17, p. 395 (2000).

11. Odian,G. - "Principles of Polymerization". John Wiley \& Sons, New York (1991).

12. Araujo,O.; Giudici,R.; Saldivar,E.; Ray,W.H. J. App. Pol. Sci. 79, p. 2360 (2001).
13. valtchev,V. Chem. Mat.. 14, p. 956 (2002).

14. Cho,J.K.; Park,B.-D.; Lee,Y.-S. Tet. Lett. 41, p. 7481 (2000).

15. arshady,R. J. Chromat.. 586, p. 181 (1991).

16. ray,W.H. J. Macromol. Sci. - Rev. Macromol. Chem.. C08, p. 01 (1972).

17. bishop,R.H. - "Learning with Labview". California, Addison-Wesley (1999).

18. Kalfas,G.; Ray,W.H. Ind. Eng. Chem. Res. 32, p. 1822 (1993).

19. Smith,W.V.; Ewart,R.H. J. Chem. Phys. 16, p. 592 (1948).

20. Kiparissides,C.; Seferlis,P.; Mourikas,G.; Morris,A.J. Ind. Eng. Chem. Res. 41, p. 6120 (2002).

21. Petzold,L.R. "A Description of DASSL: a Differential Algebraic System Solver". Sandia National Laboratories. Report \# SAND82-8637 (1982).

22. Lenzi, M. K.; Silva, F. M.; Lima, E. L.; Pinto, J. C. - J. App. Pol. Sci. 89, p. 3021 (2003).

Enviado: 25/11/03

Reenviado: 30/03/04

Aprovado: 06/04/04 